\title{
Probability Measures for Prediction in Multi-table Infomation Systems
}

\author{
R.S. Milton ${ }^{1}$, V. Uma Maheswari ${ }^{2}$, and Arul Siromoney ${ }^{2}$ \\ 1 Department of Computer Science, \\ Madras Christian College, Chennai - 600 059, India \\ 2 Department of Computer Science and Engineering, \\ College of Engineering, Guindy, \\ Anna University, Chennai - 600 025, India \\ asiro@vsnl.com, umam@annauniv.edu
}

\begin{abstract}
Rough Set Theory is a mathematical tool to deal with vagueness and uncertainty. Rough Set Theory uses a single information table. Relational Learning is the learning from multiple relations or tables. This paper presents a new approach to the extension of Rough Set Theory to multiple relations or tables. The utility of this approach is shown in classification experiments in predictive toxicology.
\end{abstract}

\section{Introduction}

Rough set theory Paw82, Paw91, KPPS99, introduced by Zdzislaw Pawlak in the early $1980 \mathrm{~s}$, is a mathematical tool to deal with vagueness and uncertainty. Rough set theory defines an indiscernibility relation that partitions the universe of examples into elementary sets. The indiscernibility relation is defined based on a single table. Relational Learning is based on multiple tables. A survey of research in Rough Sets and Relational Learning is presented in MUS04. Statistical relational learning (SRL) is a recent area of research, concerned with developing techniques to learn statistical models from relational data, and information is available at http://robotics.stanford.edu/srl/.

In this paper, a Multi-Table Information System (MTIS) is defined that extends the single information table of rough set theory to multiple information tables. The formalism used in this paper is based on tables and rows, rather than on universes and elements. Notions from the Variable Precision Rough Set model are introduced along with the notion of significant elementary sets (elementary sets in the $\beta$-positive or $\beta$-negative regions that also have a sufficiently large number of examples). This is used for prediction. An illustrative experiment in toxicology is then presented.

Another formalism for MTIS based on universes and elements is presented in MUS05. The universes correspond to the tables and the elements of the universes correspond to the rows of the tables. That formalism is applied in that paper to two special cases of MTIS. Whereas the formalism used in this paper is applicable to a general MTIS. The decision class of an unknown example 
is computed using the probability measures of the significant elementary sets in the individual tables. However, this formalism needs further study into the relationship between the probability measures of the overall system and those of the individual tables. A formal study based on probabilistic measures and distributed tables is found in [S.05].

\section{Multi-table Information System}

\subsection{Definitions}

A table $T$ is a set of rows $\left\{x_{1}, \ldots, x_{m}\right\}$ and is denoted as $T=(I, A, V, \rho)$, where $I$ is a finite set of row indices (or row identifiers), with each row index $p, 1 \leq p \leq m$, corresponding to a particular row $x_{p}$ of the table; $A$ is a finite set of attributes; $V=\bigcup_{a \in A} V_{a}$ is the set of attribute values of all attributes, where $V_{a}$ is the domain of attribute $a$; and $\rho: I \times A \rightarrow V$ is an information function such that for every row index $p \in I, \rho(p, a) \in V_{a}$ is the value of attribute $a$ in the row $x_{p}$ corresponding to the row index $p$. In other words, a row $x_{p} \in T=(I, A, V, \rho)$, $p \in I$, has the value $\rho(p, a) \in V_{a}$ for every $a \in A$. This definition is based on the definition of Rough Set Information System in Paw82.

We define a Multi-Table Information System (MTIS) as a finite set of tables denoted as $T=\left\{T_{0}, T_{1}, \ldots, T_{n}\right\}$, where $T_{0}=\left\{I_{0}, A_{0}, V_{0}, \rho_{0}\right\}$ is a decision table with two attributes in $A_{0}$, one of which is the example identifier which has a unique value for every row, and the other is a binary valued decision attribute. A similar definition is also found in Wro00. Here, each row of table $T_{0}$ corresponds to an example from the universe of examples as in the Rough Set Information System Paw82.

An example MTIS follows. Let $T=\left\{T_{0}, T_{1}, T_{2}, T_{3}\right\}$. The values of $I_{i}, A_{i}, V_{i}$, $\rho_{i}, i=0,1,2,3$ can be easily seen from the figures of the tables given below.

Table $T_{0}$
\begin{tabular}{|l|l|}
\hline drug & decision \\
\hline \hline d1 & true \\
\hline d10 & true \\
\hline d102 & true \\
\hline d110 & false \\
\hline d114 & false \\
\hline
\end{tabular}

Table $T_{3}$

\begin{tabular}{|l|l|l|}
\hline drug & property & val \\
\hline \hline d12 & salmonella & $\mathrm{p}$ \\
\hline $\mathrm{d} 205$ & chromaberr & $\mathrm{n}$ \\
\hline $\mathrm{d} 108$ & cytogen_sce & $\mathrm{n}$ \\
\hline $\mathrm{d} 1$ & cytogen_ca & $\mathrm{p}$ \\
\hline $\mathrm{d} 101$ & cytogen_ca & $\mathrm{n}$ \\
\hline
\end{tabular}

Table $T_{1}$

\begin{tabular}{|l|l|l|l|l|}
\hline drug & atom & element & type & charge \\
\hline \hline d1 & a1_1 & h & 1 & p \\
\hline d102 & a102_1 & h & 2 & p \\
\hline d1 & a1_2 & o & 1 & n \\
\hline d12 & a12_1 & o & 2 & n \\
\hline d10 & a10_1 & c & 1 & p \\
\hline d10 & a10_2 & c & 2 & p \\
\hline
\end{tabular}

Table $T_{2}$

\begin{tabular}{|l|l|l|l|}
\hline drug & atom1 & atom2 & bond-type \\
\hline \hline d12 & d12_1 & d12_2 & 7 \\
\hline d12 & d12_1 & d12_7 & 1 \\
\hline d1 & d1_14 & d1_22 & 2 \\
\hline d1 & d1_17 & d1_24 & 1 \\
\hline d101 & d101_8 & d101_9 & 2 \\
\hline
\end{tabular}




\subsection{Link Relation}

Let $T_{i}, T_{j} \in T(i \neq j)$ be two tables in a MTIS T. $T_{i}$ is said to be linked to $T_{j}$ if there exists a set of common attributes $K_{i j} \subseteq\left(A_{i} \cap A_{j}\right) \neq \emptyset$, known as link attributes, between them. Let $x_{i, p}$ be the row in table $T_{i}$ with row index $p \in I_{i}$, and $x_{j, q}$ be the row in table $T_{j}$ with row index $q \in I_{j}$. We say that $x_{i, p}$ is linked to $x_{j, q}$ through the link attributes $K_{i j}$ if $\rho_{i}(p, b)=\rho_{j}(q, b)$ for every $b \in K_{i j}$.

Formally, a binary relation $L\left(K_{i j}\right)\left(K_{i j} \neq \emptyset\right)$, called link relation, is defined between two tables $T_{i}$ and $T_{j}$ as

$L\left(K_{i j}\right)=\left\{\left(x_{i, p}, x_{j, q}\right) \in T_{i} \times T_{j} \mid \forall b \in K_{i j} . \rho_{i}(p, b)=\rho_{j}(q, b)\right.$, where $\left.p \in I_{i}, q \in I_{j}\right\}$

A row $x_{i, p}$ in table $T_{i}$ is linked to row $x_{j, q}$ in table $T_{j}$ if $\left(x_{i, p}, x_{j, q}\right) \in L\left(K_{i j}\right)$.

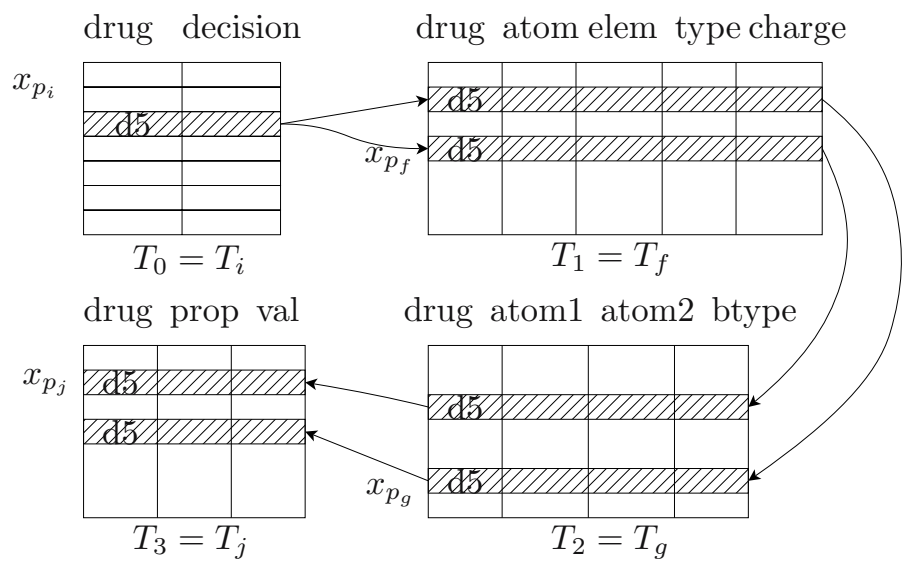

In general, a row $x_{i, p_{i}}$ in table $T_{i}$ is linked to another row $x_{j, p_{j}}$ in table $T_{j}$ iff there exist tables $T_{f}, T_{g}, \ldots, T_{h} \in T$, with rows such that $\left(x_{i, p_{i}}, x_{f, p_{f}}\right) \in L\left(K_{i f}\right)$, $\left(x_{f, p_{f}}, x_{g, p_{g}}\right) \in L\left(K_{f g}\right), \ldots,\left(x_{h, p_{h}}, x_{j, p_{j}}\right) \in L\left(K_{h j}\right)$. We say that row $x_{i, p_{i}}$ is linked through the rows $x_{f, p_{f}}, x_{g, p_{g}}, \ldots, x_{h, p_{h}}$ to row $x_{j, p_{j}}$, and the set of rows

$$
C\left(p_{i}\right)=\left\{x_{f, p_{f}}, x_{g, p_{g}}, \ldots, x_{h, p_{h}}, x_{j, p_{j}}\right\}
$$

is said to form of a combination of rows associated with $x_{i, p_{i}}$. We note that a row $x_{i, p_{i}}$ in table $T_{i}$ could have a number of such combinations of rows.

In this paper, we restrict $K_{i j}$ to a singleton set, and consider only a single link attribute between any pair of tables. In the illustration, table $T_{0}$ is linked to tables $T_{1}, T_{2}$ and $T_{3}$ through the link attribute drug.

\subsection{Elementary Sets}

Let $B_{i}$ be a subset of attributes in a table $T_{i}=\left(I_{i}, A_{i}, V_{i}, \rho_{i}\right), B_{i} \subseteq A_{i}$, such that no attribute in $B_{i}$ is either a link attribute or a primary key. A binary relation $R\left(B_{i}\right)$, called indiscernibility relation, is defined on a table $T_{i}$ as

$$
R\left(B_{i}\right)=\left\{\left(x_{i, p}, x_{i, q}\right) \in T_{i} \times T_{i} \mid \forall b \in B_{i}, \rho_{i}(p, b)=\rho_{i}(q, b), \text { where } p, q \in I_{i}\right\}
$$


We say that two rows $x_{i, p}$ and $x_{i, q}$ of table $T_{i}$ are indiscernible and belong to the same elementary set iff $\rho_{i}(p, b)=\rho_{i}(q, b)$ for $p, q \in I_{i}$ and every $b \in B_{i}$.

The elementary set of table $T_{i}$, with respect to the indiscernibility relation $R\left(B_{i}\right)$, containing the row $x_{i, p}$ is

$$
\left[x_{i, p}\right]_{R\left(B_{i}\right)}=\left\{x_{i, q} \in T_{i} \mid \forall b \in B_{i}, \rho(p, b)=\rho(q, b), \text { where } p, q \in I_{i}\right\}
$$

$\left[x_{i, p}\right]_{R\left(B_{i}\right)}$ is denoted as $\left[x_{i, p}\right]$ when $B_{i}$ is known from the context. Even though each table is partitioned into elementary sets based on the attributes in $B_{i}$, yet it continues to have all the original entries.

\subsection{Conditional Probabilities of Elementary Set}

Each row in table $T_{i} \in T, i \neq 0$, is linked to one row in the decision table $T_{0}$. Hence, each row $x_{i, p}$ in an elementary set $\left[x_{i, p}\right]$ can be considered to be associated with a decision class. For a boolean decision class, let $\left[x_{i, p}\right]^{+}\left(\left[x_{i, p}\right]^{-}\right)$ be the subset of the elementary set $\left[x_{i, p}\right]$ containing rows associated with the positive (negative) decision class.

The conditional probability that a row in the elementary set $\left[x_{i, p}\right]$ is positive is

$$
P\left(+\mid\left[x_{i, p}\right]\right)=\frac{\left|\left[x_{i, p}\right]^{+}\right|}{\left|\left[x_{i, p}\right]\right|}
$$

and $P\left(-\mid\left[x_{i, p}\right]\right)$ is similarly defined.

\subsection{Prediction}

We now consider the prediction of the decision class of a test case. We assume that the test case has a row in the decision table $T_{0}$, but does not yet have a value for the decision attribute. The associated data for this test case is introduced as rows in the other tables.

A combination of rows $C(p)=\left\{x_{1, q}, \ldots, x_{n, t}\right\}$ linked to a test case $x_{0, p}$, has one row from each of the tables with rows linked to $x_{0, p}$. Every row in the combination is linked to the test case either directly or through other rows in the combination.

Each row $x_{i, p}$ in a combination falls in an elementary set of table $T_{i}$ with the associated conditional probabilities $P\left(+\mid\left[x_{i, p}\right]\right)$ and $P\left(-\mid\left[x_{i, p}\right]\right)$. Hence, a row can be considered to have the conditional probabilities $P\left(+\mid\left[x_{i, p}\right]\right)$ and $P\left(-\mid\left[x_{i, p}\right]\right)$. We have also noted that a test case could have a number of combinations of rows.

Simple Approach: A simple approach that can be used for prediction follows. For every combination of rows corresponding to the test case with index $p_{0}$ of the decision table $T_{0}$, the product of positive conditional probabilities of rows is defined as

$$
\text { posprod }=\prod_{x \in C_{k}\left(p_{0}\right)} P(+\mid[x])
$$

Similarly, the product of negative conditional probabilities negprod is defined. If the sum of the posprods of all the combinations is greater than the sum of 
negprods of all the combinations, then the test case is predicted as positive; otherwise, it is predicted as negative. The posprod is the measure of the positive trend shown by the values of the attributes in each table. The summation of the posprod addresses the situation where a particular test case may be related to multiple rows in a table.

Significant Elementary Sets Approach: Many of the elementary sets are too small to be considered as representative of either positive or negative trend. We consider an elementary set to be a significant elementary set, if it has sufficiently large number of examples, and its $P\left(+\mid\left[x_{i, p}\right]\right) \geq \beta_{u}$ or $P\left(-\mid\left[x_{i, p}\right]\right) \leq \beta_{l}$, where $\beta_{u} \geq 0.5$ and $\beta_{l} \leq 0.5$. When $\beta_{u}=1-\beta_{l}$, we denote it as $\beta$, and note that $\beta_{l}=1-\beta$. An elementary set $\left[x_{i, p}\right]$ is said to be significant if and only if

$$
\left|\left[x_{i, p}\right]\right| \geq \alpha \wedge\left(\left(P\left(+\mid\left[x_{i, p}\right]\right) \geq \beta_{u} \vee P\left(-\mid\left[x_{i, p}\right]\right) \leq \beta_{l}\right)\right)
$$

where $\alpha$ is a user-defined parameter.

If only $k$ rows in the combination of rows fall in significant elementary sets, then we define the Positive Conditional Probability of the combination of rows as

$$
\mathcal{P}_{+}=\sqrt[k]{\prod P\left(+\mid\left[x_{i, p}\right]\right)}
$$

where $P\left(+\mid\left[x_{i, p}\right]\right.$ s are of significant elementary sets. Similarly, Negative Conditional Probability $\mathcal{P}_{-}$is defined.

A combination of rows predicts the test case as positive if $\mathcal{P}_{+} \geq \mathcal{P}_{-}$and as negative otherwise. If the number of combinations predicting the test case as positive is greater than the number of combinations predicting it as negative, the final prediction of the test case is positive; otherwise, it is negative.

\section{Application to Predictive Toxicology}

The dataset used is the Predictive Toxicology Evaluation Challenge dataset found at http://web.comlab.ox.ac.uk/oucl/research/areas/machlearn/ cancer.html. The database of compounds have been classified as carcinogens or otherwise. The challenge is to predict the carcinogenicity of previously untested chemicals. The dataset is in the form of the Multi-Table Information System described in Section 2.1.

An illustrative experiment is performed using this dataset, and the results of the ten-fold cross-validation and the summary of the results are presented in the the following tables. The results of one of the folds are not available due to an error in the experiment.

The average prediction accuracy using the simple approach is $60 \%$, and that using the significant elementary sets approach is $59.6 \%$. It is to be noted that there is no improvement in the prediction accuracy. However, the space requirement for the significant elementary sets approach is much less when compared to the simple approach. This is a difficult dataset and progol achieves only $64 \%$ even with the use of more background knowledge. 
Simple approach

\begin{tabular}{|c|c|c|c|c|}
\hline \multicolumn{2}{|c|}{ Positive } & \multicolumn{2}{|c|}{ Negative } & \multirow{2}{*}{ Accuracy } \\
\hline+ & - & + & - & \\
\hline 13 & 5 & 9 & 6 & 0.58 \\
\hline 16 & 2 & 8 & 7 & 0.70 \\
\hline 14 & 4 & 10 & 5 & 0.58 \\
\hline 14 & 4 & 13 & 2 & 0.48 \\
\hline 14 & 4 & 12 & 3 & 0.52 \\
\hline 15 & 3 & 6 & 9 & 0.73 \\
\hline 11 & 7 & 8 & 7 & 0.55 \\
\hline 18 & 0 & 13 & 2 & 0.61 \\
\hline 15 & 5 & 5 & 8 & 0.70 \\
\hline
\end{tabular}

Simple approach

\begin{tabular}{|l|c|c|c|}
\hline & Actual + & Actual - & \\
\hline Predicted + & 130 & 84 & 214 \\
\hline Predicted - & 34 & 49 & 83 \\
\hline & 164 & 133 & 297 \\
\hline
\end{tabular}

Significant elementary set approach

\begin{tabular}{|c|c|c|c|c|}
\hline \multicolumn{2}{|c|}{ Positive } & \multicolumn{2}{|c|}{ Negative } & \multirow{2}{*}{ Accuracy } \\
\cline { 1 - 3 }+ & - & + & - & \\
\hline 14 & 4 & 11 & 4 & 0.55 \\
\hline 17 & 1 & 9 & 6 & 0.70 \\
\hline 15 & 3 & 11 & 4 & 0.58 \\
\hline 15 & 3 & 10 & 5 & 0.61 \\
\hline 13 & 5 & 11 & 4 & 0.52 \\
\hline 14 & 4 & 6 & 9 & 0.70 \\
\hline 11 & 7 & 9 & 6 & 0.52 \\
\hline 17 & 1 & 15 & 0 & 0.52 \\
\hline 15 & 5 & 5 & 8 & 0.70 \\
\hline
\end{tabular}

Significant elementary set approach

\begin{tabular}{|l|c|c|c|}
\hline & Actual + & Actual - & \\
\hline Predicted + & 131 & 87 & 218 \\
\hline Predicted - & 33 & 46 & 79 \\
\hline & 164 & 133 & 297 \\
\hline
\end{tabular}

\section{Conclusions}

This paper presents an approach to learning from a Multi-Table Information System. The results of an illustrative example in toxicology are presented.

\section{References}

[KPPS99] J. Komorowski, Z. Pawlak, L. Polkowski, and A. Skowron. Rough sets: A tutorial. In S. K. Pal and A. Skowron, editors, Rough Fuzzy Hybridization: A New Trend in Decision-Making, pages 3-98. Springer-Verlag, 1999.

[MUS04] R. S. Milton, V. Uma Maheswari, and Arul Siromoney. Rough Sets and Relational Learning. LNCS Transactions on Rough Sets, Inaugural Volume, 2004.

[MUS05] R. S. Milton, V. Uma Maheswari, and Arul Siromoney. Studies on Rough Sets in multiple tables. In Dominik Ślęzak, Guoyin Wang, Marcin Szczuka, Ivo Duentsch, and Yiyu Yao, editors, The Tenth International Conference on Rough Sets, Fuzzy Sets, Data Mining, and Granular Computing (RSFDGrC 2005), pages 263-272. Springer-Verlag, 2005. LNAI 3641 (Accepted for publication).

[Paw82] Z. Pawlak. Rough sets. International Journal of Computer and Information Sciences, 11(5):341-356, 1982.

[Paw91] Z. Pawlak. Rough Sets - Theoretical Aspects of Reasoning about Data. Kluwer Academic Publishers, Dordrecht, The Netherlands, 1991.

[Ś05] D. Ślȩzak. Rough Sets and Bayes Factor. Transactions on Rough Sets, III (LNCS 3400):202-229, 2005.

[Wro00] J. Wroblewski. Analyzing relational databases using rough set based methods. In Proceedings of IPMU 2000, volume 1, pages 256-262, 2000. 